\title{
Experimental observation of second-harmonic generation and diffusion inside random media
}

\author{
Sanli Faez, ${ }^{1, *}$ P. M. Johnson, ${ }^{1}$ D. A. Mazurenko, ${ }^{1,2}$ and Ad Lagendijk ${ }^{1}$ \\ ${ }^{1}$ FOM Institute for Atomic and Molecular Physics AMOLF, Kruislaan 407, 1098 SJ Amsterdam, The Netherlands \\ ${ }^{2}$ Zernike Institute for Advanced Materials, University of Groningen, Nijenborgh 4, 9747 AG Groningen, \\ The Netherlands \\ *Corresponding author: faez@amolf.nl
}

Received September 17, 2008; revised November 21, 2008; accepted November 24, 2008; posted December 2, 2008 (Doc. ID 101717); published January 16, 2009

We have experimentally measured the distribution of the second-harmonic intensity that is generated inside a highly scattering slab of porous gallium phosphide. Two complementary techniques for determining the distribution are used. First, the spatial distribution of second-harmonic light intensity at the side of a cleaved slab has been recorded. Second, the total second-harmonic radiation at each side of the slab has been measured for several samples at various wavelengths. By combining these measurements with a diffusion model for secondharmonic generation that incorporates extrapolated boundary conditions, we present a consistent picture of the distribution of the second-harmonic intensity inside the slab. We find that the ratio $\ell_{2 \omega} / L_{c}$ of the mean free path at the second-harmonic frequency to the coherence length, which was suggested by some earlier calculations, cannot describe the second-harmonic yield in our samples. For describing the total second-harmonic yield, our experiments show that the scattering parameter at the fundamental frequency $k_{1 \omega} \ell_{1 \omega}$ is the most relevant parameter in our type of samples. () 2009 Optical Society of America

OCIS codes: $190.4400,290.4210,290.1990$.

\section{INTRODUCTION}

Optical second-harmonic generation inside inhomogeneous media has attracted much interest for biological applications, such as high-contrast microscopy [1], and for photonic applications, such as high-efficiency frequency conversion in granular nonlinear dielectrics [2]. The second-harmonic signal radiated from a random medium is also an informative probe of the fundamental and the second-harmonic intensity distribution inside the medium. Second-harmonic signals provide valuable information about multiple-scattering processes inside a random medium, which is not easily accessible by other methods. Previously, these signals have been used to study the angular, spatial, and temporal correlations inside scattering media [3-5].

Currently available first-principles theories of optical second-harmonic generation in multiple-scattering media consider only the case of the mean free path being much larger than the wavelength, far from the Anderson localization regime [6,7]. Many articles have discussed interference effects in multiple-scattering nonlinear media, such as the effect of weak localization or the enhanced forward scattering [8-11].

The efficiency of the nonlinear conversion in granular dielectrics is related to the minimized role of phasematching in such a medium. Phase-matching is essential for the efficiency of three-wave mixing processes in nonlinear materials. The optical dispersion in the nonlinear material results in a phase-mismatch between the fundamental and the higher-harmonic propagating light [12].

There are two conventional methods to overcome the phase-mismatch. The first method makes use of the fact that in birefringent and nonlinear materials, it is possible to fulfill the phase-matching condition by adjusting the crystal orientation with respect to the beam. The second method, called quasi-phase-matching, uses a periodically poled polarizability of the material. The nonlinearity of the crystal is modulated such that the extra momentum, which results from the phase-mismatch, can be transferred to the crystal. In both of these methods, the total conversion yield increases quadratically with the length of the light path inside the medium.

Recently, a novel method, called random-quasi-phasematching, has been introduced in which the crystal orientation is randomly varied along the beam path by using a powder [2]. As suggested from theory and confirmed by the experiment, for this new method, the accumulated second-harmonic energy increases linearly with the sample thickness. By using this method, a relatively high second-harmonic signal can be extracted from many semiconductor powders, which were otherwise (in their bulk form) useless for conventional methods. Using randomquasi-phase-matching, second-harmonic generation can be obtained for a larger bandwidth and acceptance angle than conventional methods.

A high second-harmonic yield has also been observed by Tiginyanu et al. [13] for strongly scattering porousGaP. Melnikov and coworkers [14,15] have shown that the second-harmonic signal in the specular-reflection direction is enhanced by orders of magnitude after anodically etching the single-crystalline $\mathrm{GaP}$ wafer. However, no comparison with a theoretical model had been provided 
for these observations. The distribution of secondharmonic light inside such a medium has also not been investigated experimentally.

In this paper, we use two complementary techniques to determine the distribution of the second-harmonic intensity inside a multiple-scattering slab. In the first experiment, the spatial distribution of second-harmonic light intensity at the side of a cleaved slab is measured. In the second experiment, the total second-harmonic radiation at each side of the slab is measured. On the macroscopic level, our experimental results confirm the predictions of the diffusion theory for distribution of the secondharmonic intensity in a multiple-scattering medium. On the microscopic level, however, the only available theoretical model by Kravtsov et al. [6], which is based on the diffusion theory, is unable to describe our experimental results. Their model suggests the ratio $\ell_{2 \omega} / L_{c}$ of the transport mean free path at the second-harmonic frequency to the coherence length as the universal parameter for the conversion yield. By performing measurements at a range of scattering strengths and frequencies we find that their model does not apply to our type of samples. Instead, our measurements show that the second-harmonic yield follows a consistent dependence on the transport mean free path at the fundamental frequency $\ell_{1 \omega}$.

In Sections 2-4 we briefly review the available theories of second-harmonic generation in random media. We present our derivation of the intensity distribution from a diffusion model with the extrapolated boundary conditions. In the experimental section we describe two separate measurement techniques that probe the distribution of second-harmonic light generation inside a highly scattering sample. Results of our experiments are then compared with predictions of the diffusion model.

\section{THEORY}

\section{A. Optical Nonlinearity in Opaque Material}

An opaque medium may also be optically nonlinear. This nonlinearity can be an intrinsic property of the bulk material or a result of the enormous interfacial area present in porous objects. The second-order nonlinearity is absent in many noncrystalline materials or crystal structures because of the presence of inversion symmetry. Relatively large nonlinearities may arise at the interfaces of these materials with others or with vacuum due to symmetrybreaking at the interface. The scientific understanding of optical nonlinear processes in strongly scattering materials is still in the preliminary phase. Some models have been developed $[6,7]$ based on the diffusion approximation, in which interference effects are assumed to be averaged out and the sample size $L$ is much larger than the transport mean free path.

In these diffusion models the incident wave at the fundamental frequency $\omega$ experiences several scattering events before leaving the random medium. Light at the second-harmonic frequency is generated during the multiple-scattering process. The propagation direction of the second-harmonic light is scrambled within one transport mean free path $\ell_{2 \omega}$, thus becoming an isotropic source of diffusive photons at the second-harmonic fre- quency. The effect of phase-mismatch between the fundamental and the second-harmonic light is negligible when the transport mean free path is much smaller than the coherence length $L_{c}(\omega) \equiv \pi /\left|2 k_{1 \omega}-k_{2 \omega}\right|$, where $k_{1 \omega}$ and $k_{2 \omega}$ are the wave vector magnitudes at the fundamental and the second-harmonic frequencies. Therefore, in a random medium that consists of grains showing a nonlinear response, the effect of constructive interference can be overcome by selecting grain sizes to be smaller than the coherence length. It has been experimentally shown that the second-harmonic yield from an equal amount of material increases with the grain size until the grain size approaches the coherence length [2].

Overcoming the destructive interference due to phasemismatch is also possible by introducing scatterers inside a homogeneous nonlinear crystal. In such a medium the fundamental and second-harmonic waves scatter differently, therefore the destructive interference of the otherwise copropagating waves does not occur, providing $\ell_{1 \omega}$, $\ell_{2 \omega} \ll L_{c}$.

\section{B. Review of Existing Theories}

Second-harmonic generation in random media has been theoretically modeled for three different systems in two reports. Here we briefly introduce and compare these models as they will be needed for comparison with our experimental results. The important prediction of these models is the magnitude of the mesoscopic [16] secondharmonic conversion rate $\Gamma$, which is defined as the second-harmonic intensity per volume, generated inside the scattering medium, per unit of fundamental energy density squared.

In the first report Kravtsov et al. [6] have considered two different systems. In the first system, scattering is introduced in a slab of nonlinear dielectric by adding a small volume fraction of pointlike scatterers. The microscopic nonlinear polarizability is assumed to be unaffected by the presence of scatterers, thus constant across the sample. We refer to this model as "homogeneous nonlinear background model." The medium can be optically dispersive, $\Delta n \equiv n_{2 w}-n_{1 w} \neq 0$. When $\Delta n \leq 0$, the conversion rate $\Gamma$ is independent of scattering parameters $k_{1 w} \ell_{1 w}$ and $k_{2 w} \ell_{2 w}$ When $\Delta n \geq 0, \Gamma$ is found to depend on $k_{1 w} \ell_{1 w}$ as

$$
\Gamma=A \varepsilon_{0} \omega n_{2 \omega} n_{1 \omega}^{2}\left[\chi^{(2)}\right]^{2} \operatorname{arccot}\left[\frac{\pi \ell_{2 \omega}}{\left(\frac{1}{2}+\frac{\ell_{2 \omega}}{\ell_{1 \omega}}\right) L_{c}}\right],
$$

where $A$ is a dimensionless prefactor, $\chi^{(2)}$ is the norm of the nonlinear polarizability of the bulk medium averaged over all directions, $L_{c}$ is the coherence length, and $\ell_{1 w}$ and $\ell_{2 w}$ are mean free paths at fundamental and secondharmonic frequencies. All parameters in Eq. (1) can be frequency dependent due to the optical dispersion of the material. In Eq. (1), $\chi^{(2)}$ and $L_{c}$ are microscopic properties of the bulk material while $\ell_{1 w}$ and $\ell_{2 w}$ are mesoscopic properties that depend on the porous structure of the material. Equation (1) predicts that as the mean free path at the second-harmonic frequency becomes small relative to the coherence length, the conversion rate should increase. The derivation of this result assumes weak multiple- 
scattering, $k_{1 w} \ell_{1 w} \gg 1, k_{2 w} \ell_{2 w} \gg 1$.

The second system that Kravtsov et al. [6] have considered is a dense powder of nonlinear grains in which the grain size is larger than the wavelength and much smaller than the mean free path. This system is similar to samples analyzed in [2]. We call this model the "nonlinear-powder model."

In the second report, Makeev and Skipetrov [7] have introduced a third system. They have modeled a suspension of colloidal particles. We refer to this model as the "nonlinear colloidal suspension model." In their model, the conversion centers are the same as the scatterers and the background medium is linear. They found that, for these suspensions, the second-harmonic intensity divided by the number of scatterers shows no explicit dependence on the multiple-scattering properties of the suspension. In their model, the second-harmonic conversion rate is given by the following simple product:

$$
\Gamma=B \rho v_{2 \omega} \Sigma_{2 \omega}
$$

where $\rho$ is the concentration of colloidal particles, $\Sigma_{2 \omega}$ is the total second-harmonic cross section of an isolated particle, $v_{2 \omega}$ is the energy velocity at the second-harmonic frequency, and $B$ is a dimensionless number.

In all three models, the nonlinearity is assumed to be so low that the distribution of fundamental diffusive photons is not affected, therefore the energy density at fundamental frequency $U_{1 \omega}(z, t)$ can be calculated by solving the diffusion equation for an optically linear multiplescattering slab.

\section{Macroscopic Distribution of the Second-Harmonic Intensity Inside an Opaque Slab}

Following the literature [6,7], we assume the source distribution of the second-harmonic diffusive photons inside the opaque material $S_{2 \omega}(\mathbf{r}, t)$ to be equal to the conversion rate $\Gamma$ times the square of the energy density of the fundamental light $U_{1 \omega}(\mathbf{r}, t)$

$$
S_{2 \omega}(\mathbf{r}, t)=\Gamma U_{1 \omega}^{2}(\mathbf{r}, t) .
$$

The exact value of $\Gamma$ depends on the model and the sample type [Eq. (1) sets an example]. Being a mesoscopic quantity, $\Gamma$ will not affect the macroscopic intensity distribution except for changing an overall prefactor.

For a slab of finite thickness, illuminated with a continuous plane wave of intensity $I_{0}$, the following set of diffusion equations can be written for the distribution of fundamental and second-harmonic diffusive-photon densities:

$$
\begin{aligned}
& D_{1 \omega} \frac{d^{2} U_{1 \omega}(z)}{d z^{2}}=-S_{1 \omega}(z), \\
& D_{2 \omega} \frac{d^{2} U_{2 \omega}(z)}{d z^{2}}=-\Gamma U_{1 \omega}^{2}(z),
\end{aligned}
$$

where $D_{1 \omega}=v_{1 \omega} \ell_{1 \omega} / 3$ and $D_{2 \omega}=v_{2 \omega} \ell_{2 \omega} / 3$ are the diffusion constants at the fundamental at the second-harmonic frequencies and $v_{1 \omega}$ and $v_{2 \omega}$ are the energy velocities in the medium. The source term $S_{1 \omega}(z)$ denotes the distribution of the diffusive light source at the fundamental frequency.
These equations must be solved together with the following boundary conditions [17]:

$$
\begin{aligned}
& U_{1 \omega}(z)-\zeta_{1 \omega}^{\prime} \frac{d U_{1 \omega}(z)}{d z}=0 \quad \text { at } z=0, \\
& U_{1 \omega}(z)+\zeta_{1 \omega}^{\prime \prime} \frac{d U_{1 \omega}(z)}{d z}=0 \quad \text { at } z=L, \\
& U_{2 \omega}(z)-\zeta_{2 \omega}^{\prime} \frac{d U_{2 \omega}(z)}{d z}=0 \quad \text { at } z=0, \\
& U_{2 \omega}(z)+\zeta_{2 \omega}^{\prime \prime} \frac{d U_{2 \omega}(z)}{d z}=0 \quad \text { at } z=L,
\end{aligned}
$$

where $\zeta_{1 \omega}^{\prime}$ and $\zeta_{2 \omega}^{\prime}$ are the extrapolation lengths at the incidence interface of the slab and $\zeta_{1 \omega}^{\prime \prime}$ and $\zeta_{2 \omega}^{\prime \prime}$ are the extrapolation lengths at the opposite interface of the slab at the corresponding frequencies.

There are various ways of formulating the source distribution at the fundamental frequency $S_{1 \omega}(z)$. A phenomenological and perhaps the most practical method [18] is to set the source at one transport mean free path from the incident interface, inside the slab $S_{1 \omega}(z)$ $=\left(v_{1 \omega} I_{0} / \ell_{1 \omega}\right) \delta\left(z / \ell_{1 \omega}-1\right)$. A natural extension [19] to the previous description considers a source term that exponentially decreases with depth, with a decay length equal to the mean free path $S_{1 \omega}(z)=\left(v_{1 \omega} I_{0} / \ell_{1 \omega}\right) \exp \left(-z / \ell_{1 \omega}\right)$. In the following calculation we use the latter description.

By integrating the diffusion Eq. (4) over an exponential source and applying boundary conditions (6) and (7) we derive, for inside of the slab,

$$
U_{1 \omega}(z)=3 I_{0}\left[\frac{\left(\ell_{1 \omega}+\zeta_{1 \omega}^{\prime}\right)\left(L+\zeta_{1 \omega}^{\prime \prime}-z\right)}{\ell_{1 \omega}\left(L+\zeta_{1 \omega}^{\prime}+\zeta_{1 \omega}^{\prime \prime}\right)}-\frac{2}{3} \exp \left(-\frac{z}{\ell_{1 \omega}}\right)\right],
$$

where the thickness $L$ of the slab is considered much larger than the mean free path, and thus terms such as $\exp \left(-L / \ell_{\omega}\right)$ are neglected.

The second-harmonic energy density is given by inserting the result of Eq. (10) in diffusion Eq. (5) followed by a double integration. The total generated second-harmonic radiation that is propagating in the $2 \pi$ spherical angle around the incidence direction is referred to as the forward radiation and denoted by $T_{2 \omega}$. The total radiation that is propagating in the opposite (backward) direction is denoted by $R_{2 \omega}$. For a slab illuminated by a plane wave, these two quantities are given by

$$
\begin{aligned}
& R_{2 \omega}=+\left.\frac{D_{2 \omega}}{v_{2 \omega}} \frac{d U_{2 \omega}(z)}{d z}\right|_{z=0}, \\
& T_{2 \omega}=-\left.\frac{D_{2 \omega}}{v_{2 \omega}} \frac{d U_{2 \omega}(z)}{d z}\right|_{z=L} .
\end{aligned}
$$

Inserting the fundamental photon-density distribution (10) in diffusion Eq. (5) and integrating yields the secondharmonic photon-density distribution inside the slab, 


$$
U_{2 \omega}(z)=\left(\zeta_{2 \omega}^{\prime}+z\right) R_{2 \omega}-\Gamma \int_{0}^{z} \int_{0}^{z_{1}} U_{1 \omega}^{2}\left(z_{2}\right) \mathrm{d} z_{2} \mathrm{~d} z_{1} .
$$

Boundary condition (8) has been applied. Applying the boundary condition (9) yields $R_{2 \omega}$. Expressions for $U_{2 \omega}$, $R_{2 \omega}$ and $T_{2 \omega}$ as functions of $\Gamma, I_{0}, L, \ell_{1 \omega}, \zeta_{1 \omega}$, and $\zeta_{2 \omega}$ were obtained by combining Eqs. (10), (11), and (13), with boundary condition (9) in MATHEMATICA. The closed forms of the answers contain many terms and are not necessary to present here for the purpose of the discussions in this paper. In completion of earlier calculations [7], we have included the extrapolated boundary conditions and exponentially decaying source term for fundamental light.

We have compared the internal distribution of secondharmonic intensity in the limiting case of vanishing extrapolation length $\zeta_{1 \omega}^{\prime}=\zeta_{2 \omega}^{\prime}=0$ with the corresponding result stated in [7] and found that their approach works well for the case of optically thick slabs with zero extrapolation length, i.e., absorbing boundaries. The effect of the finite extrapolation length is not negligible, regardless of the slab thickness.

Here we present the answer up to the first order in $\ell_{1 \omega} / L$. The first two nonzero orders suffice for most of our discussions,

$$
\begin{aligned}
R_{2 \omega}= & \frac{9 \Gamma I_{0}^{2} L}{4}\left(1+\frac{\zeta_{1 \omega}^{\prime}}{\ell_{1 \omega}}\right)^{2}\left[1+\frac{4\left(2 \zeta_{2 \omega}^{\prime}+\zeta_{2 \omega}^{\prime \prime}\right)}{3 L}\right. \\
& \left.-\frac{8 \ell_{1 \omega}^{2}\left(5 \ell_{1 \omega}+6 \zeta_{1 \omega}^{\prime}\right)}{9 L\left(\ell_{1 \omega}+\zeta_{1 \omega}^{\prime}\right)^{2}}\right]+O\left(\frac{\ell_{1 \omega}}{L}\right), \\
T_{2 \omega}= & \frac{3 \Gamma I_{0}^{2} L}{4}\left(1+\frac{\zeta_{1 \omega}^{\prime}}{\ell_{1 \omega}}\right)^{2}\left(1+\frac{4\left(2 \zeta_{2 \omega}^{\prime}+\zeta_{1 \omega}^{\prime \prime}\right)}{L}\right) \\
& +O\left(\frac{\ell_{1 \omega}}{L}\right) .
\end{aligned}
$$

As was mentioned earlier in this section, the mesoscopic conversion rate $\Gamma$ shows up only in prefactors in Eqs. (14) and (15) and does not affect the macroscopic distribution of radiation inside and around the slab. Our derivation shows that the total second-harmonic intensity is enhanced with the increasing extrapolation ratio $\zeta_{1 \omega}^{\prime} / \ell_{1 \omega}$. Physically, this enhancement is caused by the increased trapping of fundamental light inside the diffusing medium due to the interfaces.

Due to the variability of several parameters from sample to sample, it turned out to be useful to experimentally determine the ratio

$$
\eta \equiv \frac{R_{2 \omega}}{T_{2 \omega}}
$$

In theory, the dimensionless quantity $\eta$ is independent of the extrapolation ratio. Up to the the first order in $\left(\ell_{1 \omega} / L\right), \eta$ is also independent of the conversion rate $\Gamma$ and the incident intensity $I_{0}$. As reported in earlier works [3], for optically thick slabs, ratio $\eta$ converges to a constant, i.e., $\lim \eta \rightarrow 3$ when $L / \ell_{1 \omega} \rightarrow \infty$.
The most appropriate (currently available) theoretical model for our type of samples is the homogeneous nonlinear background model described in Subsection 2.B, in which the conversion rate is given by Eq. (1). The intensity of the second-harmonic radiation is dependent on several parameters. To compare any experimental result with any multiple-scattering theory, it is advantageous to separate the dependencies induced by multiple-scattering (mesoscopic) from variations that are caused by intrinsic material properties (microscopic) and from geometrical specifications of the sample (macroscopic). Therefore, for comparing the experimental results with the theory, we divide the measured second-harmonic backward radiation $R_{2 \omega}$ by the sample thickness, the incident intensity squared, and all prefactors of the arctan function in Eq. (1), which are material properties of GaP. We define the normalized yield as

$$
\gamma \equiv \frac{R_{2 \omega}}{\omega n_{2 \omega} n_{1 \omega}^{2}\left[\chi^{(2)}\right]^{2} L I_{0}^{2}},
$$

which is useful for comparing our data with the theories.

Note that in all of the above calculations, the absorption of fundamental and second-harmonic light is neglected. This assumption is justified when the absorption length of the medium is much larger than $L^{2} / \ell_{1 \omega}$, which is a justified assumption for our type of samples.

For a pulsed light source, generally, the stationary calculation is valid if $\tau_{0} \gg \tau_{D}$, where $\tau_{0}$ is the pulse duration and $\tau_{D}$ is the Thouless time, defined as $\tau_{D} \equiv L^{2} / D_{1 \omega}$. Using analytical calculations, which will be presented elsewhere, we have calculated the effect of the pulse duration on the second-harmonic intensity distribution. Although the nonstationary calculations are analytic, they contain long and complicated mathematical expressions that do not provide any intuition about the physical outcome. We found that for our specific experimental conditions, the difference between nonstationary and stationary solutions is much less than our experimental error, therefore we have presented our results only in comparison with the stationary solutions.

\section{SAMPLES AND SETUP}

\section{A. Samples}

The porous-GaP samples were etched from commercially available single crystal $n$ type gallium phosphide wafers [20]. These wafers were doped with donor atoms with a density range of $10^{18} \mathrm{~cm}^{-3}$. The fabrication procedure is as follows. One side of a $9 \times 9 \mathrm{~mm}$ piece of $\mathrm{GaP}$ wafer with a thickness of $500 \mu \mathrm{m}$ is anodically etched in sulfuric acid under a controlled electromotive force. The electric current is recorded in time. The total charge that is transported in the process is proportional to the amount of removed material, allowing for the calculation of the porosity for a known thickness of the etched layer. Different postetching processes can be used to remove the top layer in order to improve optical characterization or to increase the pore-size. We used eight samples with different values of the mean free path. These samples were previously fabricated for other optical experiments [21-23]. 
A summary of the scattering characterization properties of the investigated samples is shown in Table 1. The thicknesses were determined from scanning electron microscope images. The mean free path values were determined by the standard technique of measuring the linear total transmission. As a representative, the mean free path of sample 2 has been determined over the spectral range of 0.6 to $1.6 \mu \mathrm{m}$ (Fig. 1) by coupling a spectrometer to an integrating sphere setup. Dependence of the mean free path on the wavelength is largely due to the nanostructure of the samples. It also varies from sample to sample. The magnitude of the nonlinear susceptibility of porous-GaP for some lattice orientations is comparable to that of a commercial second-harmonic crystal, such as potassium dihydrogen phosphate (KDP). However, as $\mathrm{GaP}$ is not birefringent, it has no application for conventional methods of second-harmonic generation.

\section{B. Setup for Measuring Total Radiation}

Figure 2 shows a diagram of the integrating sphere setup that was used for measuring the total second-harmonic light that is radiated in the forward or the backward direction from a porous-GaP slab. Several gold mirrors guide the beam vertically into the entrance of a $\mathrm{TiO}_{2}$ coated integrating sphere. The sample can be laid down at the entrance for transmission measurements or placed beneath the sphere for reflection measurements. Two photodiodes are positioned at two exit holes of the integrating sphere. At one exit hole, an amplified silicon detector (PDA55a-Thorlabs) connected to an oscilloscope (DL9040L-Yokogawa) measures the second-harmonic signal after the fundamental infrared light is filtered by a cold glass filter (KG5). At the other exit hole, a germanium detector (DET10A-Thorlabs) connected to another channel of the same oscilloscope measures the scattered fundamental light. In this way, both fundamental and second-harmonic signals can be measured simultaneously.

For a second-harmonic signal to be detectable by normal silicon detectors, an incident intensity of several gigawatts per centimeters squared is essential. A long pulse at this intensity will damage GaP, therefore pulses shorter than nanoseconds with low repetition rates are needed. The light source we used was a traveling-wave collinear optical parametric amplifier of superfluorescence (TOPAS-Light Conversion) pumped with a

Table 1. Summary of Specifications of the Samples that are Analyzed in this Paper

\begin{tabular}{crccc}
\hline Tag & $L(\mu \mathrm{m})$ & $\ell_{2 \omega}(\mu \mathrm{m})$ & $\ell_{1 \omega}(\mu \mathrm{m})$ & $V_{e}(V)$ \\
\hline 1 & $35.5 \pm 1$ & $0.27 \pm 0.04$ & $2.49 \pm 0.29$ & 14.7 \\
2 & $43.7 \pm 1$ & $0.26 \pm 0.03$ & $1.85 \pm 0.29$ & 14.7 \\
3 & $23.5 \pm 1$ & $0.26 \pm 0.04$ & $2.18 \pm 0.24$ & 14.7 \\
4 & $32 \pm 1$ & $0.83 \pm 0.06$ & $2.50 \pm 0.28$ & 22.5 \\
5 & $26 \pm 1$ & $0.60 \pm 0.05$ & $2.22 \pm 0.18$ & 11.2 \\
6 & $83 \pm 3$ & $1.10 \pm 0.08$ & $4.71 \pm 0.37$ & 10.0 \\
7 & $126 \pm 4$ & $1.24 \pm 0.08$ & $3.38 \pm 0.48$ & 15.0 \\
8 & $116 \pm 3$ & $1.58 \pm 0.11$ & $5.75 \pm 0.60$ & 10.0 \\
\hline
\end{tabular}

\footnotetext{
${ }^{\mathrm{a}} V_{e}$ is the applied electromotive force during the etching process.

b The measured value of mean free paths $\ell_{2 \omega}$ and $\ell_{1 \omega}$ are presented at $\lambda_{0}$ $=0.65$ and $1.3 \mu \mathrm{m}$, correspondingly; $L$ is the thickness of the porous region.
}

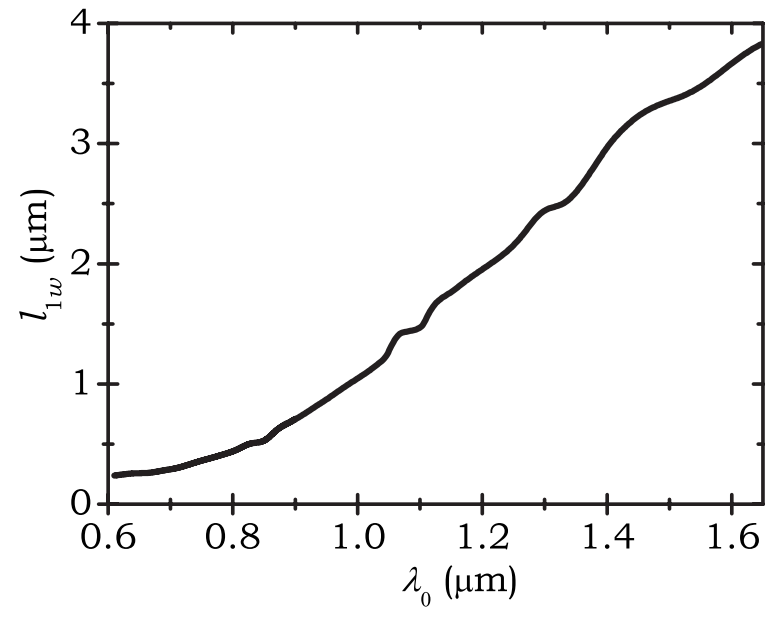

Fig. 1. Mean free path of a porous-GaP sample is wavelengthdependent. As a representative, we plot the mean free path as a function of vacuum wavelength for sample 2 . Similar curves are also obtained for all samples using total-transmission measurements.

subpicosecond laser (Hurricane-Spectra Physics) at $800 \mathrm{~nm}$ and a repetition rate of $1 \mathrm{kHz}$. The output central wavelength could be tuned continuously from 0.55 to $2.2 \mu \mathrm{m}$ with a bandwidth of $5 \mathrm{~nm}$. The pulse was transform-limited with a duration of $150 \mathrm{fs}$. The undesired frequencies are filtered out from the output beam by using a polarizer and a low-pass filter (RG850Thorlabs).

For each sample, the radiated second-harmonic light in the forward and backward directions was measured in a fundamental-wavelength range of 1.2 to $1.6 \mu \mathrm{m}$. Before each measurement, the incident power was checked by a pyroelectric head and a power meter (Ophir). The transmittance of filters and responsivities and the linearity of detectors were carefully taken into account before extracting the second-harmonic yield.

Special attention is needed to correct for the transmittance of the nonporous substrate. The total transmittance

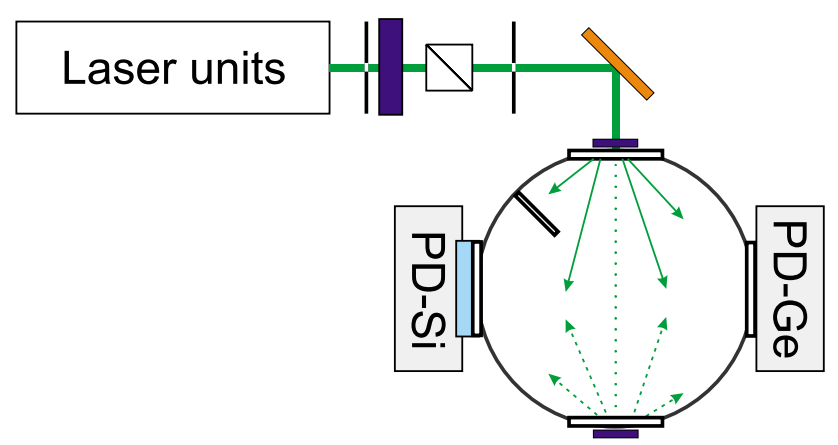

Fig. 2. (Color online) Experimental setup for measuring the total second-harmonic radiation in forward and backward directions. The porous-GaP slab is illuminated with a parallel beam of $150 \mathrm{fs}$ infrared pulses. The integrating sphere collects the fundamental and the second-harmonic light radiated in all directions from one side of the sample. The sample position is either on the top of the sphere for transmission measurements or attached beneath the sphere for reflection measurements. A silicon photodetector (PD-Si) behind a cold glass filter measures the secondharmonic signal in the visible range. In parallel, a germanium photodetector (PD-Ge) measures the transmitted fundamental light at the infrared range. 
of the porous-bulk interface depends on the refractive index mismatch at the interfaces and the directional distribution of outgoing diffuse light known as the "escape function." We used the escape function presented in [24], with an effective refractive index of $1.6 \pm 0.2$ for the porous region. This effective refractive index was measured before based on the filling fractions and escape function measurements from [22]. Using the Fresnel equations for transmittance at the substrate-air interface, the total angularly averaged transmittance of the GaP substrate for these samples has been calculated to be $0.28 \pm 0.05$, for which the magnitude of the error is mainly due to the uncertainty in the effective refractive index of the porous medium.

\section{Setup for Measuring Effusion Function}

To investigate the second-harmonic intensity distribution inside a porous-GaP sponge in greater detail, the secondharmonic radiation from the side of a cleaved porous-GaP slab was imaged under a microscope while the slab was illuminated by a parallel beam of infrared pulses at normal incidence. We refer to the diffusive energy flux at the interfaces of the random medium, which can be measured with our method as the "effusion function." The measurement of the effusion function is sensitive to details of the distribution of the second-harmonic source inside the slab. To test this sensitivity, we calculated the secondharmonic effusion function for a half-slab numerically and compared it with the bulk second-harmonic energy distribution in a slab, predicted by Eq. (13). We observed that the second-harmonic effusion pattern approximates the bulk second-harmonic intensity distribution well. This result suggests that one can get an immediate qualitative sense of the second-harmonic energy distribution from the observation of the second-harmonic effusion function.

The effusion microscopy setup is shown in Fig. 3. The incident beam in this setup is a Gaussian infrared beam of $3 \mathrm{~mm}$ in diameter with its center aimed toward the edge of the sample. A cold glass filter in front of the CCD camera blocks the fundamental light so that only the visible second-harmonic light is captured.

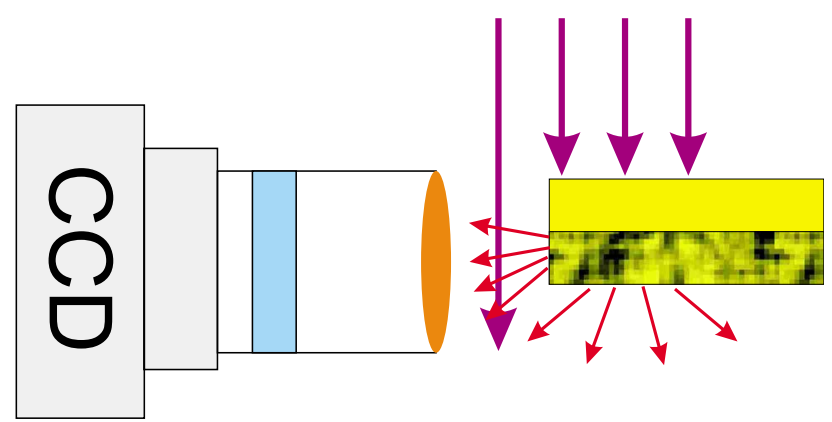

Fig. 3. (Color online) Experimental setup for effusion microscopy measurements. The sample consists of a thin porous and highly scattering layer laying on top of a transparent substrate. The CCD camera images the second-harmonic signal that is radiated from the narrow cross section of the porous part of the sample while it is illuminated by a parallel beam of infrared pulses.

\section{RESULTS}

\section{A. Dependence on Incident Power}

At an incident wavelength of $1.2 \mu \mathrm{m}$, where the efficiency is the highest, the dependence of the second-harmonic yield on the incident power is measured for several samples in the forward direction. The fundamental power is linearly proportional to voltage $V_{0}$ of the silicon detector. The second-harmonic power is linearly proportional to voltage $V_{2}$ of the germanium detector. The measured relation between output voltages is plotted in Fig. 4. From this plot a consistent power-law dependence between incident and second-harmonic powers is evident for these samples. The result of fitting shows a power law $P_{2 \omega} \propto P_{0}^{\alpha}$ with $\alpha=1.87 \pm 0.03$. In a second-order nonlinear process the second-harmonic intensity is proportional to the square of the incident intensity. The observed deviation of the experimentally measured $\alpha$ from 2.0 may be a sign of nonlinear (three photon) absorption inside the porousGaP.

\section{B. Effusion Function at Second-Harmonic Frequency}

The microscopy setup described in Subsection 3.C has been used to capture the effusion function at the secondharmonic frequency. As the incident beam size is much larger than the slab thickness, the effusion pattern is laterally (parallel to the substrate) invariant. Roughness of the section due to its porosity and cleaving-defects can cause fluctuations in the observed intensity. The intensity distribution is averaged in the lateral direction. The experimental result is compared with our numeric calculation of the diffusion model in Fig. 5. The bulk distribution of the second-harmonic intensity is also plotted for comparison.

The measured second-harmonic intensity distribution qualitatively agrees with the diffusion model. The intensity has a maximum around one third of the slab thickness, as was predicted theoretically in [7]. The largest deviation is observed near the edges, which we attribute to

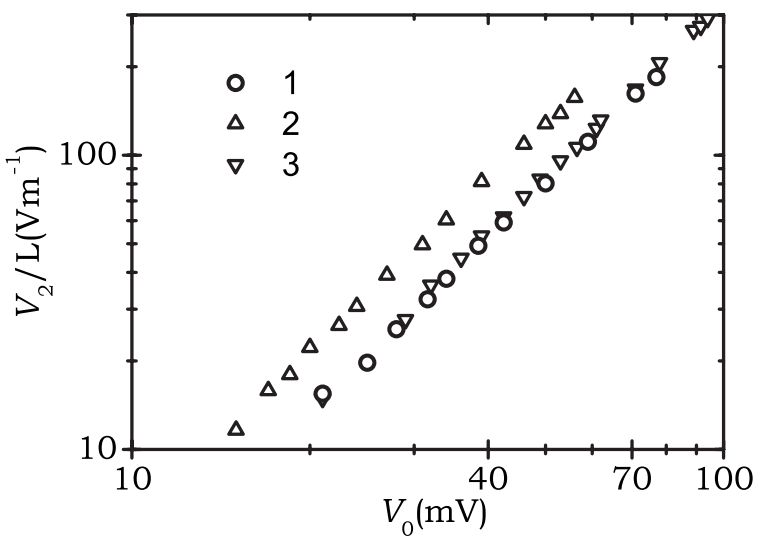

Fig. 4. Backward-radiated second-harmonic signal $V_{2}$, detected by the silicon detector, is scaled by the sample thickness and plotted for three of the samples versus the signal detected by a germanium detector $V_{0}$ in bilogarithmic scales. The secondharmonic power is proportional to $V_{2}$ and shows a power-law dependence (exponent $=1.87 \pm 0.03$ ) on the incident power, which is proportional to $V_{0}$. Numbers in the legend indicate sample tags as introduced in Table 1. 


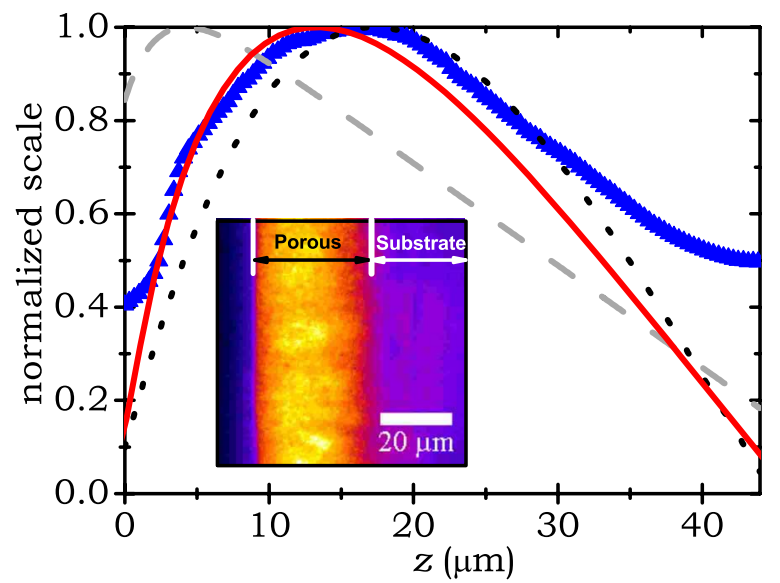

Fig. 5. (Color online) Inset, micrograph of the second-harmonic effusion intensity from a section of the porous-GaP slab while it is illuminated with a parallel beam of infrared laser pulses from the left. Brighter regions indicate higher effusion of secondharmonic light. Outset, the measured second-harmonic intensity is averaged parallel to the substrate and its peak is normalized to 1 . The result of the experiment (symbols) is plotted versus the position inside the sample and is compared with the prediction of the stationary diffusion model (solid curve), found from the numeric calculation with no adjustable fitting parameters. Our theoretical value for distribution of the second-harmonic intensity in the bulk, which is presented in Subsection 2.C, is shown by the dotted curve. The theoretical fundamental-frequency intensity distribution inside the slab (dashed curve) is plotted for comparison.

the stray second-harmonic light that is leaving the other interfaces of the slab behind the imaging plane.

\section{Second-Harmonic Intensity Distribution in Far-Field} The measured value of $\eta$, defined by Eq. (16), has been plotted versus the optical thickness of samples at various fundamental wavelengths in Fig. 6. The calculated $\eta$ in the framework of the stationary diffusion model according to Eq. (16) is also plotted for comparison. We find a good agreement between measurement and theory for most of

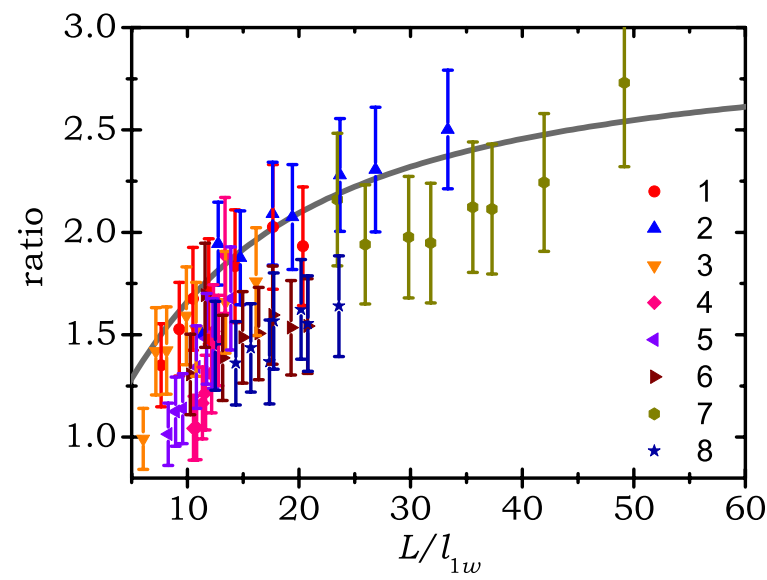

Fig. 6. (Color online) Ratio $\eta$ between total second-harmonic light measured in the backward and the forward direction is plotted versus the optical thickness $L / \ell_{1 \omega}$ for various wavelengths and samples. The stationary diffusion prediction from Subsection 2.C is plotted as a solid curve. We find a good agreement between theory and measurements. Numbers $1-8$ in the legend correspond to the sample numbers introduced in Table 1. the samples. Large error bars are mainly due to the uncertainty in the effective refractive index of the porous medium.

\section{Second-Harmonic Yield}

At several incident frequencies, we have measured the total second-harmonic intensity radiated from the sample. Extracting the absolute value of the conversion rate from the second-harmonic intensity measurements is limited by a large systematic shift that arises from uncertainties in the response function of the setup. However, this systematic shift does not affect the relative values. Therefore for testing theoretical models, we consider relative trends rather than the absolute values.

To check whether the homogeneous nonlinear background model of Kravtsov et al. [6] can describe our data, the normalized yield $\gamma$ defined by Eq. (17) has been plotted versus the ratio $\ell_{2 \omega} / L_{c}$. This plot is shown in Fig. 7(a) for all samples and measured wavelengths. For this plot the optical dispersion relation and the nonlinear polarizability of the bulk GaP has been taken from measurements of [25] and calculations of [26], respectively. Our measurements show a trend opposite to the homogeneous background model [6]. For an individual sample, the yield increases with an increasing mean free path relative to the coherence length. The incremental trend is different from sample to sample, which indicates that $\ell_{2 \omega} / L_{c}$ is not the universal parameter for describing the conversion rate in our kind of samples.

For the normalized yield plotted versus the scattering parameter at the fundamental frequency $k_{1 \omega} \ell_{1 \omega}$, most of the measurement points for all the samples are close to a single curve. The consistent trend of an increasing yield with decreasing $k_{1 \omega} \ell_{1 \omega}$ occurs both when comparing different wavelengths in a single sample and when comparing different samples at the same wavelength. We have fitted these data to a power-law function and found an average exponent of $-2.0 \pm 0.3$.

\section{CONCLUSION}

Using a microscopy technique, we have measured the second-harmonic intensity at the side of a cleaved-slab during its illumination with a Gaussian beam. We have observed that the internal distribution of secondharmonic intensity predicted by the diffusion model agrees with the experiment. The total intensity radiated in the backward direction and the backward-forward ratio of the second-harmonic intensity has also been measured for a number of samples. The measured backwardforward ratios show good agreement with the results of the diffusion model.

For describing our distribution measurements, we have presented a diffusion model for second-harmonic intensity distribution in a strongly scattering nonlinear material in which the diffused fundamental intensity is converted into its second-harmonic via the process of degenerate two-photon mixing. The second-harmonic light also diffuses in the scattering medium. The internal distribution and the outgoing intensity in forward and backward directions of a slab are derived based on the diffusion equation for light and extrapolated boundary conditions. As 


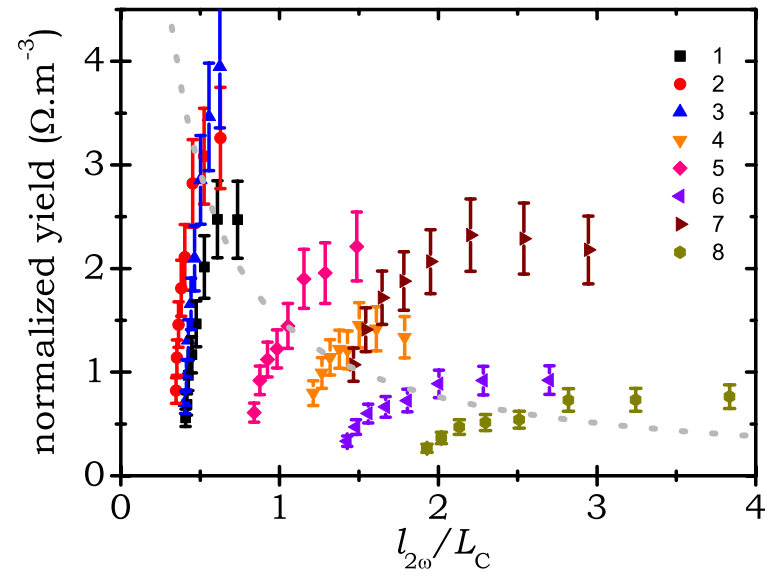

(a)

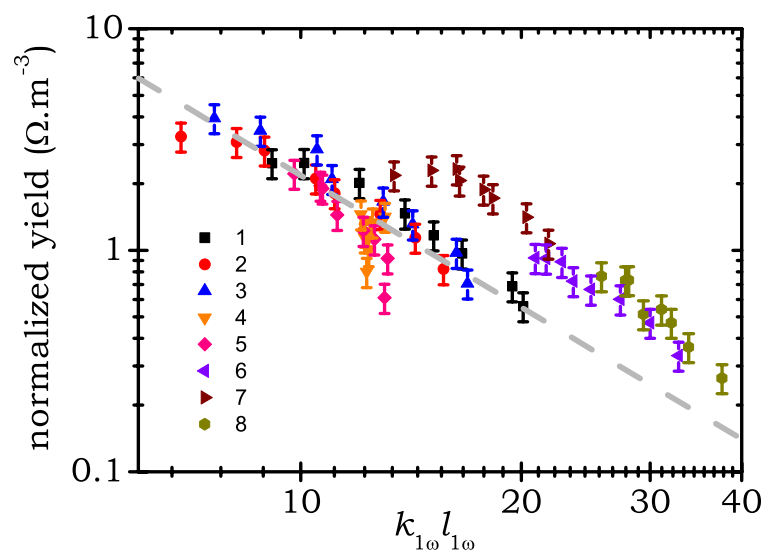

(b)

Fig. 7. (Color online) (a) Second-harmonic normalized yield as defined in Eq. (17) is plotted versus the ratio of the mean free path at the second-harmonic frequency to the coherence length $\ell_{2 \omega} / L_{c}$ for various wavelengths and samples. The normalized yield is defined as the total second-harmonic intensity generated in the backward direction divided by the square of incident intensity and the thickness of the slab and normalized for frequency dependent material properties of GaP. The dotted curve shows the value calculated from the theoretical model of Kravtsov et al. [6] plotted for comparison. No agreement has been found between their theory and our measurements. Numbers 1-8 in the legend correspond to the sample numbers introduced in Table 1. (b) Same data of (a) is plotted versus the scattering strength at the fundamental frequency. The overall trend can be described by a power-law relation, $\gamma \propto\left(k_{1 \omega} \ell_{1 \omega}\right)^{\beta}, \beta=$ $-2.0 \pm 0.3$, which is shown by the dashed curve.

was reported previously [6,7], the diffusion theory predicts that in the slab geometry, the total generated second-harmonic intensity increases linearly with the thickness of the slab and depends quadratically on the extrapolation ratio at the fundamental frequency. As a result of the diffusion model, the ratio $\eta$ between the second-harmonic radiated intensity in backward and forward directions is independent of the mesoscopic conversion efficiency and is only given by the slab-geometry and boundary conditions. Therefore, $\eta$ provides a useful test for the diffusion model, irrespective of the microscopic details. For a sufficiently thick slab, $\eta$ approaches 3 as predicted by the diffusion theory. This result has been confirmed by our measurements.
Although current diffusion models describe well the distribution of the second-harmonic intensity, the overall conversion rate cannot be described by the available theories. We have found a consistent power-law dependence between the second-harmonic yield and the scattering parameter at the fundamental frequency, $\gamma \propto\left(k_{1 \omega} \ell_{1 \omega}\right)^{\beta}$, with an exponent of $\beta=-2.0 \pm 0.3$.

\section{ACKNOWLEDGMENTS}

We thank Willem Vos for sharing equipment, which was essential for our experiments and Otto Muskens for helpful collaboration and discussion. This work is part of the research program of the "Stichting voor Fundamenteel Onderzoek der Materie," which is financially supported by the "Nederlandse Organisatie voor Wetenschappelijk Onderzoek."

\section{REFERENCES}

1. P. J. Campagnola and L. M. Loew, "Second-harmonic imaging microscopy for visualizing biomolecular arrays in cells, tissues and organisms: optical imaging," Nat. Biotechnol. 21, 1365-1360 (2003).

2. M. Baudrier-Raybaut, R. Haïdar, Ph. Kupecek, Ph. Lemasson, and E. Rosencher, "Random quasi-phasematching in bulk polycrystalline isotropic nonlinear materials," Nature 432, 374-376 (2004).

3. J. F. de Boer, A. Lagendijk, R. Sprik, and S. Feng, "Transmission and reflection correlations of secondharmonic waves in nonlinear random media," Phys. Rev. Lett. 71, 3947-3950 (1993).

4. T. Ito and M. Tomita, "Speckle correlation measurement in a disordered medium observed through second-harmonics generation," Phys. Rev. E 69, 036610 (2004).

5. M. Tomita, "Coherence coupling effect in a space- and time-resolved, nonlinear-correlation measurement in a multiple-scattering medium,” J. Opt. Soc. Am. B 22, 537-546 (2005).

6. V. E. Kravtsov, V. M. Agranovich, and K. I. Grigorishin, "Theory of second harmonic generation in strongly scattering media," Phys. Rev. B 44, 4931-4942 (1991).

7. E. V. Makeev and S. E. Skipetrov, "Second-harmonic generation in suspensions of spherical particles," Opt. Commun. 224, 139-147 (2003).

8. V. E. Kravtsov and V. M. Agranovich, "Nonlinear backscattering from opaque media," Phys. Rev. B 43 13691-13694 (1991).

9. A. Heiderich, R. Maynard, and B. A. van Tiggelen, "Coherent backscattering in nonlinear media," Opt. Commun. 115, 392-400 (1995).

10. A. G. Mal'shukov and G. D. Mahan, "Nonlinear forward scattering of light in opaque media," Phys. Rev. B 57, 7701-7704 (1998).

11. T. Wellens, B. Grémaud, D. Delande, and C. Miniatura, "Coherent backscattering of light by nonlinear scatterers," Phys. Rev. E 71, 055603 (2005).

12. R. W. Boyd, Nonlinear Optics (Academic, 2003).

13. I. M. Tiginyanu, I. V. Kravetsky, J. Monecke, W. Cordts, G. Marowsky, and H. L. Hartnagel, "Semiconductor sieves as nonlinear optical materials," Appl. Phys. Lett. 77, 2415-2417 (2000).

14. V. A. Melnikov, L. A. Golovan, S. O. Konorov, D. A. Muzychenko, A. B. Fedotov, A. M. Zheltikov, V. Yu. Timoshenko, and P. K. Kashkarov, "Second-harmonic generation in strongly scattering porous gallium phosphide," Appl. Phys. B 79, 225-228 (2004).

15. L. A. Golovan, V. A. Melnikov, K. P. Bestemyanov, S. V. Zabotnov, V. M. Gordienko, V. Yu. Timoshenko, A. M. Zheltikov, and P. K. Kashkarov, "Disorder-correlated enhancement of second-harmonic generation in strongly 
photonic porous gallium phosphide," Appl. Phys. B 81, 353-356 (2005).

16. A mesoscopic property in multiple-scattering of light [after M. C. W. van Rossum and Th. M. Nieuwenhuizen, "Multiple scattering of classical waves: microscopy, mesoscopy, and diffusion," Rev. Mod. Phys. 71, 313-371 (1999)] refers to those dependencies that are induced by multiple-scattering. In accordance, parameters that are dependent on intrinsic material properties are called microscopic and properties that reflect geometrical specification of the sample including its size are called macroscopic.

17. A. Ishimaru, Wave Propagation and Scattering in Random Media (Academic, 1978).

18. E. Akkermans, P. E. Wolf, and R. Maynard, "Coherent backscattering of light by disordered media: analysis of peak line shape," Phys. Rev. Lett. 56, 1471-1474 (1986).

19. D. J. Durian, "Penetration depth for diffusing-wave spectroscopy," Appl. Opt. 34, 7100-7105 (1995).

20. B. H. Erné, D. Vanmaekelbergh, and J. J. Kelly, "Morphology and strongly enhanced photoresponse of GaP electrodes made porous by anodic etching," J. Electrochem. Soc. 143, 305-314 (1996).

21. F. J. P. Schuurmans, D. Vanmaekelbergh, J. van de Lagemaat, and A. Lagendijk, "Strongly photonic macroporous GaP networks," Science 284, 141-143 (1999).

22. J. G. Rivas, "Light in strongly scattering semiconductores-diffuse transport and Anderson localization," Ph.D. dissertation (Universiteit van Amsterdam, 2002).

23. B. P. J. Bret, "Multiple light scattering in porous gallium phosphide," Ph.D. dissertation (Universiteit Twente, 2005).

24. M. U. Vera and D. J. Durian, "Angular distribution of diffusely transmitted light," Phys. Rev. E 53, 3215-3224 (1996).

25. D. E. Aspnes and A. A. Studna, "Dielectric functions and optical parameters of $\mathrm{Si}, \mathrm{Ge}, \mathrm{GaP}, \mathrm{GaAs}, \mathrm{GaSb}, \mathrm{InP}$, InAs, and InSb from 1.5 to $6.0 \mathrm{eV}$," Phys. Rev. B 27, 985-1009 (1983).

26. Z. H. Levine, "Optical second harmonic susceptibilities: frequency-dependent formulation with results for $\mathrm{GaP}$ and GaAs," Phys. Rev. B 49, 4532-4538 (1994). 